\author{
Gdańsk 2018, Nr. 39 \\ https://doi.org/10.26881/sgg.2018.39.14 \\ Wilfried Kürschner \\ Universität Vechta
}

\title{
Taxierende Attribute
}

Im folgenden Beitrag wird auf eine bestimmte Sorte adjektivischer Attribute aufmerksam gemacht. Sie treten im Strukturschema „Adjektiv + Numerale + Substantiv (Maßangabe/Zählgröße)“ auf. Mit ihnen gibt der Sprecher dem Hörer vor, wie er den angesprochenen Sachverhalt auf einer Skala von „niedrig“ bis „hoch“, „wenig“ bis „viel“ oder dergleichen eingeschätzt, taxiert sehen möchte. Neben solchen skalaren Attributen weisen andere Belege übliche beschreibende Attribute auf. Die Belegsammlung, alphabetisch geordnet nach den vorkommenden Adjektiven, findet sich im Anhang.

Schlüsselwörter: Adjektiv, Attribut, Einschätzung, Taxierung

Attributes of taxation. - The following paper deals with a special kind of adjectival attributes, so-called attributes of taxation. They appear in the structure "adjective + numeral + noun (of measure)". Their purpose is to direct the hearer's taxation of the measurement implied along the scale of "low" to "high", of "few" to "many" etc. Apart from these scalar attributes, our corpus (in the appendix) exhibits "normal" descriptive ones.

Keywords: adjective, attribute, estimation, taxation

In den vergangenen Jahren habe ich mehr oder weniger systematisch Belege notiert, die das Phänomen aufweisen oder aufzuweisen scheinen, um das es hier geht. Aus dieser Sammlung - sie besteht aus gut hundert Sätzen oder kurzen Texten - stammen die meisten Beispiele, aber beginnen möchte ich mit einem selbstkonstruierten Beispielpaar:
(1a) Die Temperatur beträgt milde $10 \mathrm{Grad}$.
(1b) Die Temperatur beträgt kühle $10 \mathrm{Grad}$.

Es zeigt hoffentlich deutlich, worum es hier geht und was mich zum Beschreibungsbegriff „taxierend“ bewogen hat. Dieser Begriff wird meines Wissens in der Literatur zum Attribut nicht verwendet, wie überhaupt festzustellen ist, dass das Attribut als Erscheinung der Syntax und der Semantik noch nicht in seiner Gesamtheit bearbeitet worden ist.

Zurück zu den beiden konstruierten Beispielen. Man hat so etwas Ähnliches wahrscheinlich schon einmal im Wetterbericht gehört, im Radio oder im Fernsehen, wo das früher mit großem Ernst behandelte Thema jetzt locker-flockig präsentiert wird. In gedruckter Form, etwa in den Zeitungen, finden sich solche Formulierungen eher seltener, woraus sich ergibt, dass unser taxierendes Attribut eine Angelegenheit der kolloquialen gesprochenen Umgangssprache ist, die aber sortenspezifisch in die geschriebene Sprache Einzug gehalten hat. 
Am besten zeigt sich die Funktion der im Eingangsbeispiel vorkommenden taxierenden Attributsadjektive mild und kühl, wenn wir sie weglassen. Dann lautet unser Satz

(1c) Die Temperatur beträgt 10 Grad.

Das ist eine nüchterne, man könnte auch sagen: „objektive“ Aussage, die sich auf das Thermometer mit seiner Maßeinheit Grad bezieht. Die Werte sind dort auf einer Skala angeordnet, in unserem Beispielsatz auf der Stelle $10 \mathrm{Grad}$. So weit, so gut, so objektiv. Bei den beiden taxierten Fassungen dieses objektiven Sachverhaltssatzes treten nun die Größen Sprecher, Hörer und Kommunikationssituation hinzu. Der Sprecher nimmt den Hörer gewissermaßen bei der Hand und gibt ihm zu verstehen, dass die Gradzahl in der gegebenen Wetterlage, in der er sich befindet, und angesichts dessen, was üblicherweise „für die Jahreszeit“" zu erwarten ist, als mild und damit angenehm oder eben als kühl und damit unangenehm einzustufen ist. Der Sprecher übernimmt sozusagen die Interpretationshoheit, was vom Hörer nicht unbedingt als freundlicher Akt gedeutet wird - er kann sich ja selber einen Reim auf die Temperatur machen und sie so oder so empfinden, oder sie kann ihm auch gleichgültig sein. Da braucht er nicht unbedingt eine Vorgabe. Taxierende Attribute haben also etwas Dominierendes, zumindest Steuerndes an sich. Andererseits können sie, wie wir an späteren Beispielen sehen werden, auch willkommene Einschätzungshilfen sein, oder kann jeder auf Anhieb sagen, ob der Preis für eine Pizza Marinara in Höhe von 4,50 Euro als hoch oder niedrig (oder eben normal) einzuschätzen ist? „Objektiv“ kann man das nicht sagen, es kommt auf die Umstände an, den Ort, das Lokal, die Größe, die Qualität, das Preisniveau im Ganzen - und da liest man nun gern, dass der Preis in einer der Null-Kilometer-Trattorien in Rom schlappe 4,50 Euro beträgt, also ziemlich niedrig ist:

(2) In einer zertifizierten Null-Kilometer-Trattoria [in der die Speisenbestandteile direkt aus der Umgebung stammen] kann man eine Portion Pasta al Pomodoro für sechs Euro bekommen, eine Pizza Marinara für schlappe 4,50 Euro [...]. (Frankfurter Allgemeine Zeitung [= F.A.Z.], 2012-08-20)

Eine Betrachtungs- und Redeweise aufgreifend, die aus Weinrichs Konzeption der Instruktionssemantik in Erinnerung ist, können taxierende Attribute als spezielle Art semantischer Anweisungen des Sprechers an den Hörer beschrieben werden mit der allgemeinen Form, dass er, der Hörer, einem Wert auf einer Skala - bisher hatten wir Temperaturgrade und Geldbeträge - eine bestimmte relative Position zuschreiben soll, „hoch“ oder „,niedrig“.

Wir haben es der syntaktischen Form nach mit Gruppen des Typs

(3) Attributsadjektiv + Numerale + Substantiv der Maßangabe/Zählgröße $(+$ Substantiv der Bezugsgröße)

zu tun. Das Adjektiv bezieht sich syntaktisch auf das Substantiv der Maßangabe bzw. der Zählgröße, semantisch auf die Kombination aus Numerale + Substantiv. Das Substantiv muss wohl immer im Plural stehen (in dieser Kombination aber nicht immer Pluralform haben). Der Singular scheint ausgeschlossen zu sein, wie die folgenden, wiederum konstruierten Sätze nahelegen:

(4a) Ein Glas Bier kostet stolze fünf Euro.

(4b) Ein Glas Bier kostet * schlappe einen Euro. 
(4c) Ein Glas Bier kostet einen ?schlappen Euro.

(5a) Die Temperatur beträgt erträgliche zwei Grad.

(5b) Die Temperatur beträgt * erträgliches ein Grad.

(5c) Die Temperatur beträgt erträgliche null Grad.

Das letzte Beispiel zeigt übrigens, dass die Zahlangabe null wie ein Plural zu funktionieren scheint. Eine Bemerkung am Rande: Die Zahl ein funktioniert wohl immer wie ein Singular, selbst wenn der Sache nach unzweifelhaft eine Mehrzahl gemeint ist: tausendundeine Nacht, nicht Nächte.

Zurück zur Strukturformel „Attributsadjektiv $+\underline{\text { Numerale }+ \text { Maßsubstantiv/Zählgröße }}$ (+ Substantiv der Bezugsgröße)“, wie wir sie etwa in Satz 6 haben:

(6) In der letzten Umfrage des Deutschen Sauna-Bundes stellten beachtliche 78 Prozent der Befragten heraus, nur selten oder gar nicht an grippalen Infekten zu erkranken. (Kneipp-Journal, $01-02 / 2010)$

Die Formel ist, wie nicht anders zu erwarten, nicht zementiert, sondern erweiterbar, wie Satz 7 und 8 zeigen, wo das Attribut modifiziert wird:

(7) In Mecklenburg-Vorpommern sind es 60 Prozent, die über diese Verstehenskompetenz [des Niederdeutschen] verfügen, im Stadtstaat Bremen sind es durchaus überraschende 58 Prozent. (Frerk Möller: Plattdeutsch im 21. Jahrhundert. Bestandsaufnahme und Perspektiven. [...] Leer: Schuster, 2009: 29)

(8) Der Aktienkurs von Google war am ersten Handelstag um vergleichsweise geringe 18 Prozent gestiegen. (F.A.Z., 2011-05-21)

Die Taxierung kann natürlich auch mit anderen Mitteln als dem adjektivischen Attribut signalisiert werden. Der Autor von Satz 7 stellt die Sachlage, offensichtlich auf stilistische Varianz bedacht, in Satz 9 und Satz 10 mithilfe von Umstandsbestimmungen dar: „In Bremen haben - durchaus überraschend - 23 Prozent angegeben [...]“, „Erstaunlicherweise sind es in Bremen 42 Prozent aller Befragten [...].“

(9) In Bremen haben - durchaus überraschend - 23 Prozent angegeben, die Sprache in diesem Maße [,sehr gut/gut"] sprechen zu können, in Mecklenburg-Vorpommern sind es 23 [sic] Prozent. (Möller 2009: 33)

(10) Erstaunlicherweise sind es in Bremen 42 Prozent aller Befragten, die sich entweder am Befragungstag oder „in der vergangenen Woche“ auf Platt unterhalten haben [...]. (Möller 2009: 35)

In meiner Sammlung findet sich ein schöner Beleg, in dem die Einschätzung eines Wertes, seine Taxierung, nicht in Form eines kondensierten Attributs erfolgt, sondern in einem Text ausführlich begründet wird. Es geht dort um die Rückforderung von Boni, die Bankmitarbeiter erhalten haben, und zu der eine Umfrage bei Finanzinstituten durchgeführt wurde. Von 63 Instituten geben 17 Prozent an, Rückforderungen vorgenommen zu haben. Sind 17 Prozent nun ein hoher oder ein niedriger Wert?

(11) Im vergangenen Jahr haben etliche Banken bereits an Mitarbeiter ausgezahlte Boni wegen Ergebnisberichtigungen oder Dienstvergehen zurückgefordert. In einer Umfrage der Unternehmensberatung Mercer unter 63 internationalen Finanzinstituten berichteten 17 Prozent der befragten 
Banken, dass sie Boni oder andere Vergütungskomponenten von Mitarbeitern zurückgefordert hätten. In der Fachsprache wird dieser Vorgang „Clawback“ genannt. Clawbacks wurden im Zuge der jüngsten Finanzkrise in vielen Banken eingeführt, um die Mitarbeiter davon abzuhalten, hohe Risiken mit der Absicht einzugehen, kurzfristig die variable Vergütung zu steigern. Knapp zwei Drittel der befragten Banken haben inzwischen in ihren Vergütungssystemen verankert, dass Rückforderungen möglich sind. Zu den häufigsten Auslösern zählen Verstöße gegen den internen Verhaltenskodex und Kompetenzüberschreitungen. Nach Einschätzung der Berater sind die 17 Prozent kein besonders hoher Wert - Mercer spricht von einer „relativ geringen Nutzung“" der Rückforderungsmöglichkeit. Dies liege wahrscheinlich daran, dass dieses Instrument noch vergleichsweise neu sei. (F.A.Z., 2012-08-25)

Im Text wird am Ende erläutert, dass „die 17 Prozent kein besonders hoher Wert“, also ein ziemlich niedriger sind. Diese Einschätzung hätte gleich am Anfang durch Formulierungen wie „[...] berichteten nur“ oder „lediglich 17 Prozent der befragten Banken [...]“ zum Ausdruck gebracht werden können.

Die Partikeln nur und lediglich - auch an gerade einmal wäre zu denken - sind die Standardmittel, wenn ein Wert als relativ niedrig eingestuft werden soll.

(12) Internet und Telefon [...] alles für nur 19,90€/Monat im 1. Jahr und danach für günstige 17,90/ Monat. (Werbung)

(13) Während in Deutschland 211 Milligramm Antibiotikum pro Kilogramm erzeugte tierische Biomasse eingesetzt werden, sind es in Dänemark gerade einmal 43 Milligramm. (Oldenburgische Volkszeitung (Vechta) $[=O V], 2014-04-01)$

(14) In Durm, Mustafi, Goretzka, Hahn, Meyer und Volland hat der Bundestrainer gleich sechs Profis mit insgesamt null Einsatzzeit in der Nationalelf nominiert - und Ginter als Nummer sieben bringt es auf gerade ein Minütchen. (F.A.Z., 2014-05-09)

Alternativen dazu sind taxierende Adjektive wie niedrig oder schlapp (wie in Satz 2 oben).

Offenbar spielen Werte, die an den oberen und unteren Extrempunkten von Skalen liegen, wahrnehmungsmäßig eine besondere Rolle. Zu ihrer sprachlichen Charakterisierung in taxierenden Attributen dienen spezielle Adjektive, unter denen das Adjektiv stolz in unserer Sammlung besonders häufig erscheint. So ist von „stolzen 45 Jahren Betriebszugehörigkeit“ die Rede:

(15) Das neue Jahr hat bei Big Dutchman mit zwei ganz besonderen Arbeitsjubiläen begonnen: Heinz Kühling blickt auf stolze 45 Jahre Betriebszugehörigkeit beim Calveslager Stalleinrichter zurück. $(O V, 2010-02-06)$

von „stolzen 34 Jahren Erfahrung“:

(16) Nach seinem Referendariat kam er 1977 ans Humboldt-Gymnasium. Das sind mittlerweile stolze 34 Jahre Erfahrung. (SpaZz. Das Magazin für Ulm und Neu-Ulm, Juli 2011)

von „stolzen 49 Euro“ für eine Flasche Balsamico:

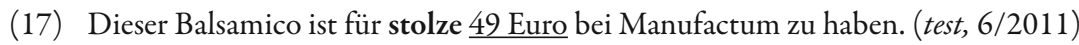

von „stolzen 195 Euro“ für einen Haarglätter:

(18) Der sehr gute Haarglätter „Braun Satin Hair 7“ kostet 63 Euro und das gute Kultgerät „ghd IV styler" stolze 195 Euro. (test, 6/2011) 
von „stolzen 2000 Fans im Werder-Stadion“:

(19) Ein Testspiel des Zweiligisten FC St. Pauli beim Bezirksligisten Sparta Werlte (4:0) lockte jetzt stolze 2000 Fans ins Werder-Stadion. (OV, 2012-07-09)

- wenn es sich um stolze Fans gehandelt hätte, hätte es übrigens 2000 stolze Fans heißen müssen. In Satz 20, der diese Reihenfolge „Numerale + Adjektiv + Substantiv (Zählgröße)“ aufweist, liegt bei 800 stolze Euro wohl eine Vertauschung vor:

(20) Für 600 Euro Honorar im Monat zu jobben stellt für sie [= Verena] eine tolle Herausforderung dar - die optimale Mitarbeiterin. [...] worauf Verena die Buchhaltung komplett übernimmt - für nunmehr 800 stolze Euro im Monat. (F.A.Z., 2011-08-04)

Die Angabe stolz bezieht sich auf diverse Größen: Zeiträume, Preise, Anzahlen. Dies hat mit der Grundbedeutung des Adjektivs stolz bestenfalls im metaphorischen Sinn zu tun, weshalb für diese Bedeutungsvariante eine Eintragung in den Wörterbüchern zu erwarten ist. Im „Duden-Universalwörterbuch“ ( $\left.{ }^{8} 2015\right)$ ist zu lesen: „(im Hinblick auf Anzahl, Menge, Ausmaß) erheblich, beträchtlich; als ziemlich hoch empfunden; beeindruckend." Als typische Fügungen werden genannt: „eine stolze Summe, eine stolze Zahl, ein stolzer Preis“ und auch für unseren Zusammenhang „stolze 21 Prozent Gewinn“. Stilistisch wird diese Verwendung von stolz als umgangssprachlich markiert. In Wahrigs „Deutschem Wörterbuch“ ('2011) wird die Sonderstellung des taxierenden stolz nicht so eindeutig klar gemacht, sondern lediglich in der Fügung „ein stolzer Preis“ mit der Angabe „ein recht hoher Preis“ und der stilistischen Charakterisierung „figurativ, umgangssprachlich“ erwähnt. Bei Wahrig wird auch das Antonym zu stolz, das schon erwähnte schlapp, gar nicht als Besonderheit erkannt, sehr wohl aber wieder im „Universalduden“. Dort wird die als „salopp“ markierte Verwendung von schlapp definiert mit ,gerade (mal) eben; knapp, gerade ausreichend“. Das Beispiel lautet: „Momentan gibt es nur schlappe zwei Prozent Zinsen aufs Sparbuch" (woraus übrigens die Zeitgebundenheit von Wörterbuchbeispielsätzen erhellt: Heute würde man fast von „stolzen zwei Prozent Zinsen aufs Sparbuch" sprechen).

Einen weiteren Fall eines in übertragener Bedeutung gebrauchten Adjektivs findet wir im Beleg 21. Dort ist von „satten 81 Prozent“ befragten Amerikanern die Rede, die einen bestimmten Umstand ihres Lebens gern ändern würden.

(21) Satte 81 Prozent [der in einer Umfrage eines Kondomherstellers befragten Amerikaner] würden nach Wegen suchen, ihr Sexualleben aufregender zu gestalten. (OV, 2012-07-14)

(22) BMW hat ein Gewichtsproblem. Das 240i Gran Coupé ist satte 242 Kilogramm schwerer als der vergleichbare Mercedes CLS 350. (Auto, Motor und Sport, 20/2012)

Das Attribut unterstützt hier die durch die Zahlenangabe ohnehin nahegelegte Ansiedlung des Wertes im oberen Skalenbereich. Im „Universalduden“ ist dies wieder ordnungsgemäß vermerkt und exemplifiziert. Stilistische Angabe zu satt: „umgangssprachlich“, Bedeutungsumschreibung: ,ansehnlich“, Verwendungsbeispiele: „satte Gewinne; es kostet satte 580 Euro; satte $16 \%$ Sollzinsen“. Wahrig schneidet wiederum deutlich schlechter ab. Dort werden für satt vier Bedeutungsvarianten genannt; unser satt gehört wahrscheinlich zu Variante 3, „umgangssprachlich“, mit der Definition „gut, reichlich bemessen“. Ein passendes Beispiel 
steht wie bei den übrigen Varianten nicht direkt dahinter, sondern ist in einem Bündel undifferenzierter Kollokationen verborgen: „satte zwei Millionen.“

Schließlich noch zu müde, wie es in Beispiel 23 vorliegt, wo von „müden sechs Prozent“ die Rede ist und der Wert somit als niedrig eingestuft wird:

(23) Normalerweise liegt der Marktanteil der ARD da [= bei den Jugendlichen] bei müden sechs Prozent. $(O V, 2012-01-25)$

Im „Universalduden“ findet sich kein entsprechendes Beispiel, wohl aber die Redewendung von der müden Mark (jetzt auch dem müden Euro), die man für etwas nicht auszugeben bereit ist. Es handelt sich dabei um eine negativ-polare Lexikoneinheit (keine müde Mark). In Satz 24 erscheint „ein müder Dollar“ in positiv-polarem Kontext; mir scheint der Satz ungrammatisch zu sein:

(24) Und dass sie [= Jodie Foster] ihr Privatleben so effektiv abgeschirmt hat, eröffnete ihrem Nichtsnutz von Bruder die Möglichkeit, mit einem Buch über die gemeinsame frühe Kindheit einen müden Dollar zu verdienen. (F.A.Z., 2012-08-17)

Neben diesen Spezialfällen stolz, schlapp, satt und müde und vielleicht einigen mehr stehen „,normale" Adjektive zur Verfügung. Die meisten von ihnen stufen einen Wert als auf der dazugehörigen Skala hoch angesiedelt ein: Satz 25: ,unglaubliche 2,7 Millionen Liter Wasser pro Sekunde“:

(25) In Hufeisenform stürzen hier [an den Niagarafällen] unglaubliche 2,7 Millionen Liter Wasser pro Sekunde auf einer Breite von 670 Metern etwa 54 Meter in die Tiefe. (Reiseprospekt „Kanada: Indian Summer“, Marco Polo Reisen, 2010)

Satz 26: „unglaubliche 850 Meldungen“:

(26) Unglaubliche 850 Meldungen. Ponyclub Dinklage hat an drei Turniertagen rappelvolles Programm (Rundschau am Mittwoch [Vechta], 2011-03-09, Überschrift und Unterüberschrift)

Satz 27: „eindrucksvolle 24.900 Quadratmeter“:

(27) In dieser grünen Oase von eindrucksvollen 24.000 Quadratmetern finden sich Wiesen, alter Baumbestand, ein Teich und die beliebte Bocciabahn. (Prospekt „Augustinum Bonn“, ca. 2010)

Satz 28: „schmerzhafte 2,5 Prozentpunkte“:

(28) So würde zum Beispiel ein Ölpreisschock mit Preisen zu 150 Dollar je Barrel das Wachstum der Weltwirtschaft um schmerzhafte 2,5 Prozentpunkte bremsen. (Focus Money, Nr. 16/2011, 2011-04-13)

So verhält es sich auch in Beleg 29, wo das Gewicht eines tragbaren Computers mit „angenehmen 1,32 Kilogramm" beschrieben wird:

(29) Das Gewicht [des Computers] beträgt angenehme 1,32 Kilogramm [...]. (F.A.Z., 2011-11-08)

Es geht hier allerdings nicht um die Gewichtsskala, sondern um einen hohen Wert auf der Komfortskala seines Trägers.

Wir müssen nun noch auf einige der unklaren Fälle aus der Sammlung zu sprechen kommen. Nehmen wir den folgenden Beleg:

(30) [...] beläuft sich der Preis auf empfohlene 1400 Euro. (F.A.Z., 2011-11-08) 
Die syntaktische Strukturformel ist zweifelsohne erfüllt. Aber dennoch würden wir bei empfoblen nicht gern von einem taxierenden Attribut im bislang gemeinten Sinn sprechen. Wir erhalten ja keine Auskunft darüber, wo der Preis auf einer Skala von billig bis teuer anzusiedeln ist, wie es etwa bei „satten“ oder „günstigen 1400 Euro“ der Fall wäre. Es liegt hier offenbar ein ganz normales beschreibendes Attribut vor. So auch beide Male in Beleg 31:

(31) Für jede Rasur der Grashalme gibt es außerdem verschiedene Maschinen, je nachdem wie kurz die Rasenfläche sein muss. Das reicht dann von samtigen drei Millimetern rund um die Löcher bis zu recht haarigen 50 Millimetern am Platzrand. (OV, 2011-09-15)

Einen ähnlichen Fall haben wir beim ersten Attribut im folgenden Beleg: „hübsche 1,60 Meter“: beschreibend, taxierend hingegen ist das zweite Attribut, „imposante 2,06 Meter“, zu verstehen.

(32) In kompletter Teamstärke traten die Oyther Volleyball-Damen und die Basketballer vom SC Rasta zur Bürgerparty an. Mit VfL-Libero Sabine Neumann trafen hübsche 1,60 Meter auf imposante 2,07 Meter. Die misst Rasta-Center Marcus King-Stockton. (OV, 2010-09-27, Bildunterschrift)

Die nächste Verwendung von bübsch ist wieder im taxierenden Sinn zu verstehen (so wie auch in einem Ausdruck wie ein hübsches Sümmchen), wohl auch mit gewollter Anspielung auf das Aussehen des gemeinten Models:

(33) Das sieht man am anschaulichsten an der Spitze der Verdiensthierarchie. Bei den Männermodels steht da laut Forbes-Liste Sean O’Pry mit 1,5 Millionen Dollar Einnahmen im Jahr 2013, die Frauen haben hingegen Gisele Bündchen mit hübschen 42 Millionen Dollar. (F.A.Z., 2014-03-21)

Bei einigen Belegen bin ich mir unsicher, wie sie zu deuten sind, unter anderem Nummer 34:

(34) In Washington selbst zahlt man für die renommierteste Uni, die Georgetown University, lockere 50.000 Dollar pro Jahr. Und selbst für eine staatliche Uni legt man noch gute 10.000 Dollar hin. (SpaZz. Das Magazin für Ulm und Neu-Ulm, Juli 2011)

Den ersten Satz mit den „lockeren 50.000 Dollar“" würde ich eher so formulieren, dass locker als Umstandsbestimmung fungiert: „Man zahlt locker 50.000 Dollar im Jahr“. Die attributive Stellung lässt sich vielleicht durch Analogie zu „stolze 50.000 Dollar“ erklären. Für die „guten 10.000 Dollar“ habe ich allerdings keine Erklärung. Ist vielleicht gut 10.000 Dollar gemeint, also „etwas mehr als 10.000 Dollar“? Allerdings finden sich weitere derartige Belege in der Sammlung:

(35) Gute 50 Kilometer von Hermannstadt entfernt empfängt die Stadt an der Kokel [= Mediasch] den Besucher auf dem Hauptplatz mit entspannter Atmosphäre [...]. (Hermannstädter Zeitung, Nr. 2250, 2011-09-16)

(36) Die Schüssel hat einen Durchmesser von gerade einmal guten zwölf Zentimetern, ist aber ein Jahrtausend alt und stammt aus der Song-Dynastie (960 bis 1279). (OV,2013-Mrz-21)

(37) Josef Hürkamp ist und bleibt der Radler. Gute 10000 Kilometer [...] hat er Jahr für Jahr wohl zurückgelegt und dabei selbst weiteste Strecken in Kauf genommen. (OV,2014-03-12)

Abschließend noch die beiden folgenden Belege im Vergleich: 
(38) Als bescheidene Kapitale der Weinregion fungiert der träge, ganze eintausendsechshundert Einwohner zählende Marktflecken Olonzac. (F.A.Z., 2011-08-25)

(39) Ein Plattenbau nach dem anderen wuchs aus dem Boden und schließlich auch das Sonnenblumenhaus in Lichtenhagen, ein Riese unter den Plattenbauten, der ganze elf Etagen hochragt. (F.A.Z., 2012-08-22)

Beide enthalten das Attribut ganz. In Satz 38 zeigt es einen geringen Wert an („ganze 1600 Einwohner" in einer Kapitale), in Satz 39 dagegen, wie auch aus dem Kontext hervorgeht („ein Riese unter den Plattenbauten“), einen hohen: „ganze elf Etagen“. Legt man die Wörterbuchangaben zu dieser Verwendung von ganz zugrunde - „Universalduden“: „, (in Verbindung mit einer Kardinalzahl) (ugs.) nicht mehr als: das Buch hat ganze fünf Euro gekostet“, Wahrig: ,nur, nicht mehr als“ (,ich besitze noch ganze drei Euro“) -, wäre 39 abweichend. Hier scheint allerdings ein lexikalischer Wandel im Gange, wie die folgenden Belege zeigen. Wie aus den Kontexten hervorgeht, signalisiert ganz hier ebenfalls keinen niedrigen, sondern einen hohen Wert:

(40) Einsteigen und sparen. Beim Kauf des neuen Multivan LIFE erhalten Sie jetzt nicht nur einen Preisvorteil von bis zu 3.200 Euro. Sie bekommen außerdem ganze 1.000 Euro Rabatt pro Kind. (Lokalhelden. Das Stadtmagazin für Vechta, H. 7, Frühjahr 2013)

(41) Das Gusserzeugnis [= die Glocke] wirkt mit einem Durchmesser von 59 Zentimetern zwar relativ klein, bringt jedoch ganze 120 Kilogramm auf die Waage. (OV, 2014-03-12)

(42) Bis heute fanden sich über „Bauer sucht Frau“ nach Angaben von RTL mehr als 20 glückliche Paare. „15 Mal wurden Heiratsanträge gestellt und ganze elf Mal wurde die Liebe vor dem Traualtar besiegelt. Sechs Kinder krönen die beeindruckende Erfolgsbilanz", frohlocken die Macher der Sendung. (OV, 2013-10-21)

(43) Johannes Sigismund Stapf von der Juristischen Fakultät ist der Rektor mit den meisten Amtszeiten - zwischen 1701 und 1741 wurde er ganze 22-mal gewählt. (uni leben)

Man darf gespannt sein, wann dieser Wandel Eingang in die Wörterbücher findet.

Anhang: Liste der die Strukturformel „Attributsadjektiv + Numerale + Substantiv der Maßangabe/Zählgröße (+ Substantiv der Bezugsgrößße)" erfüllenden Belege

\section{aberwitzig}

Der selige Mercedes W 140 wurde für ein Leergewicht von 2,1 Tonnen gescholten - und heute wiegt ein Audi 57 aberwitzige 2018 Kilogramm. (Auto, Motor und Sport, 20/2012, 2012-09-06)

\section{angenehm}

Das Gewicht [sc. des Computers] beträgt angenehme 1,32 Kilogramm [...]. (F.A.Z., 2011-11-08)

Die Temperaturen sollen bei angenehmen $20 \mathrm{Grad}$ Celsius liegen. (OV, 2014-07-12) 
ansehnlich

Die meisten Aktienfonds enthalten einmalige Ausgabeaufschläge von 5 Prozent und jährliche Verwaltungsgebühren von 2 Prozent, die sich im Laufe von drei Jahrzehnten auf ansehnliche 121000 Euro beziehungsweise ein Viertel des Endvermögens vor Steuern summieren. (F.A.Z., 2014-03-29)

\section{atemberaubend}

Nicht alles ist Schulte-Noelle geglückt, doch auch sein teuerster Fehler, die Übernahme der Dresdner Bank für atemberaubende 24 Millionen Euro, ist ihm von den AllianzAktionären längst verziehen. (F.A.Z., 2012-08-25)

\section{attraktiv}

Im Rahmen der Frühjahrs-Sonderzinsaktion gelten für ADAC-Mitglieder ab 30.04.2014 attraktive 4,30 \% effektiver Jahreszins bei einer Laufzeit von bis zu 60 Monaten. (ADAC Motorwelt, Heft 5/2014)

\section{beachtlich}

In der letzten Umfrage des Deutschen Sauna-Bundes stellten beachtliche 78 Prozent der Befragten heraus, nur selten oder gar nicht an grippalen Infekten zu erkranken. (Kneipp-Journal, 01-02/2010)

Noch-Chef [der Deutschen Flugsicherung] Kaden kassierte 2010 beachtliche $\underline{436000}$ Euro. (Der Spiegel, 8/2012, 2012-02-18)

\section{beeindruckend}

Das neue Jahr hat bei Big Dutchman mit zwei ganz besonderen Arbeitsjubiläen begonnen: Heinz Kühling blickt auf stolze 45 Jahre Betriebszugehörigkeit beim Calveslager Stalleinrichter zurück. Beeindruckende 40 Jahre ist Heinrich Fraas bei Big Dutchman tätig. $(O V, 2010-02-06)$

\section{bescheiden}

Der [Faltrollstuhl] Caneo 200 wiegt selbst relativ bescheidene 27 Kilogramm. (F.A.Z., 2012-07-10)

\section{beträchtlich}

Mit „beträchtlichen“drei Prozent, also rund 1,65 Millionen Euro, sei auch das Finanzamt Vechta als eines von 57 niedersächsischen Finanzämtern [an den aus Kapitalerträgen bei niedersächsischen Finanzämtern hinterzogenen 161,7 Millionen Euro] beteiligt, so Dornieden [= Vorsteher des Vechtaer Finanzamtes]. (OV, 2010-03-19)

\section{biblisch}

Heute wird der große deutsche Schriftsteller Siegfried Lenz biblische 85 Jahre alt. $(O V, 2011-03-17)$ 


\section{eindrucksvoll}

In dieser grünen Oase von eindrucksvollen 24.000 Quadratmetern finden sich Wiesen, alter Baumbestand, ein Teich und die beliebte Bocciabahn. (Prospekt „Augustinum Bonn“, ca. 2010)

\section{empfindlich}

Der CCS-Anschluss (am Wagen) kostet überdies empfindliche 590 Euro extra. (F.A.Z., 2014-03-04)

\section{empfoblen}

[...] beläuft sich der Preis auf empfohlene 1400 Euro. (F.A.Z., 2011-11-08)

endlos

Nun muss Christians Boot vom Felsen gezogen werden, bevor es zerreißt. Mit zwei langen Seilen ringen wir dem Fluss das Boot eine endlose halbe Stunde später endlich ab. (F.A.Z., 2008-06-19)

\section{enttäuschend}

Enttäuschende 8,4 Prozent haben die Grünen bei der Bundestagswahl geholt [...]. (Süddeutsche.de, 2013-10-19)

\section{erheblich}

Und seine [= Gottschalks] Premiere am Montag wollten auch erhebliche 4,34 Millionen [sc. Zuschauer] sehen. $(O V, 2012-01-25)$

\section{erlaubt}

Ein Motorradfahrer wurde [...] mit 229 statt der erlaubten 100 Stundenkilometer gemessen. $(O V, 2013-10-12)$

\section{erschreckend}

Bei einem weiteren Szenario haben wir [...] mit Preissteigerungen gerechnet: jährlich mit 5 Prozent für Gas und Strom, 7 Prozent für Öl und mit moderaten 3,5 Prozent für Pellets. Die Folgen sind vor allem beim ungedämmten Haus spürbar. [...] Wird es mit Öl beheizt, klettern die jährlichen Gesamtkosten im Durchschnitt der kommenden 15 Jahre

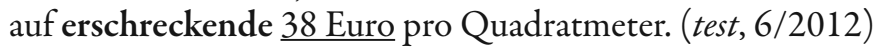

\section{erstaunlich}

„Auch mit diesen Grenzen kann das Herunterladen von einem Gigabyte Daten über die Grenzen hinweg noch erstaunliche 700 Euro kosten", sagt Beuc-Generaldirektorin Monique Goyens. $(O V, 2012-06-30)$ 


\section{federleicht}

Eine der kleinsten Drohnen der Welt. [...] Dieser Winzling wiegt federleichte $13 \mathrm{~g}$. (Katalog Pro-Idee, Frühjahr 2018)

frei

Bei Dropbox hingegen ist die Startoption mit 2 Gigabyte knapp bemessen, kann aber ... auf freie 18 Gigabyte gesteigert werden. (F.A.Z., 2013-10-01)

\section{fürstlich}

Seine [= Chaplins] Gage waren fürstliche 175 Dollar die Woche. (OV, 2014-04-16) ganz

Als bescheidene Kapitale der Weinregion fungiert der träge, ganze eintausendsechshundert Einwohner zählende Marktflecken Olonzac. (F.A.Z., 2011-08-25)

Das Desinteresse [an Marieluise Fleißer in Ingolstadt] besteht hingegen auch heute noch fort. Ganze vier Besucher haben sich an diesem Sonntag in der Kupferstraße 18 [ihrem Geburtshaus] eingefunden. (F.A.Z., 2013-06-12)

Ein Plattenbau nach dem anderen wuchs aus dem Boden und schließlich auch das Sonnenblumenhaus in Lichtenhagen, ein Riese unter den Plattenbauten, der ganze elfEtagen hochragt. (F.A.Z., 2012-08-22)

Einsteigen und sparen. Beim Kauf des neuen Multivan LIFE erhalten Sie jetzt nicht nur einen Preisvorteil von bis zu 3.200 Euro. Sie bekommen außerdem ganze 1.000 Euro Rabatt pro Kind. (Lokalhelden. Das Stadtmagazin für Vechta, Heft 7, Frühjahr 2013)

Das Gusserzeugnis [= die Glocke] wirkt mit einem Durchmesser von 59 Zentimetern zwar relativ klein, bringt jedoch ganze 120 Kilogramm auf die Waage. (OV, 2014-03-12)

Bis heute fanden sich über "Bauer sucht Frau“ nach Angaben von RTL mehr als 20 glückliche Paare. „15 Mal wurden Heiratsanträge gestellt und ganze elf Mal wurde die Liebe vor dem Traualtar besiegelt. Sechs Kinder krönen die beeindruckende Erfolgsbilanz", frohlocken die Macher der Sendung. (OV, 2013-10-21)

Johannes Sigismund Stapf von der Juristischen Fakultät ist der Rektor mit den meisten Amtszeiten - zwischen 1701 und 1741 wurde er ganze 22-mal gewählt. (uni leben)

\section{gefuiblt}

Gefühlte 50 Flaschen SlivOVic werden in dem Film geleert [...]. (Hermannstädter Zeitung, Nr. 2250, 2011-09-16)

Die süddeutsche Variante des hochdeutschen „nicht" ist das „net“. Stattdessen ist beim BR [Bayerischen Rundfunk] in gefühlten 98 Prozent das nördliche „nich“ zu hören [...] (F.A.Z., 2012-09-05)

\section{gering}

Der Aktienkurs von Google war am ersten Handelstag um vergleichsweise geringe 18 Prozent gestiegen. (F.A.Z., 2011-05-21) 
geschätzt

Der Wert der Beute beläuft sich auf geschätzte 15000 Euro. (OV, 2013-09-16)

Das Angebot an Leuchtmitteln im Baumarkt verteilt sich auf geschätzte zehn Regalmeter. (test, 10/2013)

\section{großartig}

Sie [= die verkaufte Auflage] stieg im zweiten Quartal 2016 gegenüber dem Vorjahreszeitraum um großartige 17 Prozent auf 28.246 Exemplare. (Werbebrief „Junge Freiheit“, 2016-09)

günstig

Internet und Telefon [...] alles für nur 19,90 €/Monat im 1. Jahr und danach für günstige 17,90/Monat. (Werbung)

Wer mehr braucht, kann Kapazitäten dazubuchen, beispielsweise weitere $100 \mathrm{~GB}$ für

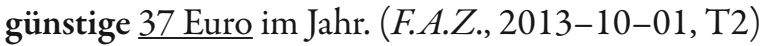

gut

In Washington selbst zahlt man für die renommierteste Uni, die Georgetown University, lockere 50.000 Dollar pro Jahr. Und selbst für eine staatliche Uni legt man noch gute 10.000 Dollar hin. (SpaZz. Das Magazin für Ulm und Neu-Ulm, Juli 2011)

Gute 50 Kilometer von Hermannstadt entfernt empfängt die Stadt an der Kokel [= Mediasch] den Besucher auf dem Hauptplatz mit entspannter Atmosphäre [...]. (Hermannstädter Zeitung, Nr. 2250, 2011-09-16)

Die Schüssel hat einen Durchmesser von gerade einmal guten zwölfZentimetern, ist aber ein Jahrtausend alt und stammt aus der Song-Dynastie (960 bis 1279). (OV,2013-03-21)

Josef Hürkamp ist und bleibt der Radler. Gute 10000 Kilometer [...] hat er Jahr für Jahr wohl zurückgelegt und dabei selbst weiteste Strecken in Kauf genommen. ( $O V$, 2014-03-12)

\section{haarig}

Für jede Rasur der Grashalme gibt es außerdem verschiedene Maschinen, je nachdem wie kurz die Rasenfläche sein muss. Das reicht dann von samtigen drei Millimetern rund um die Löcher bis zu recht haarigen 50 Millimetern am Platzrand. (OV, 2011-09-15)

\section{handwarm}

Bollert der Ofen aber im Ausnahmefall lange Zeit auf $250 \mathrm{Grad}$, können die Temperaturen in der Mitte der Glasscheibe auch schon einmal auf mehr als $90 \mathrm{Grad}$ steigen. Das tut richtig weh. Doch es geht auch kühler. Bosch HBG73B550 und die Siemens-Baugleichheit begnügen sich zum Beispiel mit handwarmen $30 \mathrm{Grad}$. (test, 9/2012)

\section{happig}

Fast alle Anbieter haben aber eine zusätzliche SMS-Flat im Repertoire. Viele verlangen dafür happige 12,95 Euro monatlich. (test, 9/2012) 


\section{bübsch}

In kompletter Teamstärke traten die Oyther Volleyball-Damen und die Basketballer vom SC Rasta zur Bürgerparty an. Mit VfL-Libero Sabine Neumann trafen hübsche $\underline{1,60}$ Meter auf imposante 2,07 Meter. Die misst Rasta-Center Marcus King-Stockton. ( $\mathrm{OV}$, 2010-09-27, Bildunterschrift)

Das sieht man am anschaulichsten an der Spitze der Verdiensthierarchie. Bei den Männermodels steht da laut Forbes-Liste Sean O'Pry mit 1,5 Millionen Dollar Einnahmen im Jahr 2013, die Frauen haben hingegen Gisele Bündchen mit hübschen 42 Millionen Dollar. (F.A.Z., 2014-03-21)

\section{imposant}

In kompletter Teamstärke traten die Oyther Volleyball-Damen und die Basketballer vom SC Rasta zur Bürgerparty an. Mit VfL-Libero Sabine Neumann trafen hübsche 1,60 Meter auf imposante 2,07 Meter. Die misst Rasta-Center Marcus King-Stockton. (OV, 2010-03-27, Bildunterschrift)

\section{kärglich}

Das Gepäckabteil misst etwas kärgliche 400 Liter, die Hybridversion hat gar nur 310 Liter Stauvolumen. (F.A.Z., 2013-10-15)

\section{kümmerlich}

[...] führte der US-Konzern Apple im Jahr 2010 für einen Auslandsgewinn von rund 13 Milliarden Dollar nur kümmerliche 120 Millionen Dollar Steuern ab. [Microsoft überwies auf Auslandserträge von gut 15 Milliarden Dollar lediglich 1,7 Milliarden an den Fiskus.] (Der Spiegel, 46/2012, 2012-11-12,72)

\section{lächerlich}

In den siebziger Jahren wuchs daneben [ = neben der „Kampfbahn Rote Erde“ in Dortmund] das mit für heutige Verhältnisse lächerlichen 31 Millionen Mark errichtete „Westfalenstadion" " [...]. (F.A.Z., 2012-08-24)

Man muss regelmäßig fast mitweinen, wenn im fernen Täbris ein noch entfernterer Onkel des Teppichhändlers verblichen ist und die wunderbaren Teppiche aus der Region nunmehr für lächerliche 159,99 statt 1599,99 Euro verscherbelt werden müssen. (F.A.Z., 2014-03-21)

\section{lang}

Wer über die Beatles redet, muss auch über Zahlen sprechen. „Please Please Me“, das erste Album der Band, hielt sich lange 30 Wochen an der Spitze der britischen Charts, der Nachfolger "With the Beatles" 21 Wochen. (Der Spiegel, Nr. 21/2010, 2010-05-22)

\section{locker}

In Washington selbst zahlt man für die renommierteste Uni, die Georgetown University, lockere 50.000 Dollar pro Jahr. Und selbst für eine staatliche Uni legt man noch gute 10.000 Dollar hin. (SpaZz. Das Magazin für Ulm und Neu-Ulm, Juli 2011) 


\section{mangelhaft}

Mangelhafte 17 Punkte [bei einem Autowerkstättentest] bekam der Bosch-Dienst Frischholz in der Hansestadt Hamburg. (test, 9/2012)

mau

Bei der Europawahl 2009 lag die Beteiligung im Kreis noch bei mauen 42 Prozent. $(O V, 2014-05-24)$

\section{mild}

Die Temperaturen pendeln sich auf „für die Jahreszeit normale bis milde neun bis $14 \mathrm{Grad}$ ein", wie Stefan Bach vom Deutschen Wetterdienst (DWD) in Offenbach sagte. $(O V, 2014-03-17)$

\section{mittelmäßig}

In Deutschland dagegen, wo man in den letzten Tagen in weiten Teilen des Landes Temperaturen von mittelmäßigen $\underline{20}$ und nachts nur schlotternden zehn $\underline{\text { Grad }}$ maß, soll nach einem Bericht des Deutschen Wetterdienstes vom Dienstag die erste Hälfte des Jahres 2011 dennoch zu warm ausgefallen sein - nämlich um 1,5 Grad wärmer, als es hierzulande typisch ist. (F.A.Z., 2011-07-27)

\section{moderat}

Bei einem weiteren Szenario haben wir [...] mit Preissteigerungen gerechnet: jährlich mit 5 Prozent für Gas und Strom, 7 Prozent für Öl und mit moderaten 3,5 Prozent für Pellets. Die Folgen sind vor allem beim ungedämmten Haus spürbar. [...] Wird es mit Öl beheizt, klettern die jährlichen Gesamtkosten im Durchschnitt der kommenden 15 Jahre auf erschreckende 38 Euro pro Quadratmeter. (test, 6/2012)

Es sind bei gleichem Preis (moderate 300 Euro) vier statt zwei Gigabyte Arbeitsspeicher an Bord [...]. (F.A.Z., 2014-03-04)

\section{müde}

Normalerweise liegt der Marktanteil der ARD da [sc. bei den Jugendlichen] bei müden sechs Prozent. $(O V, 2012-01-25)$

niedrig

Kühlten wir die Kühlkammer versuchsweise von 25 auf niedrige $10 \mathrm{Grad}$ ab, sank der Stromverbrauch der meisten Geräte um rund die Hälfte. (test, 7/2012)

\section{rasant}

Ein HD-Film käme damit in etwa 45 Minuten ins Haus. [...] Es geht noch schneller. Mit den rasanten 128000 Kilobit pro Sekunde von Tele Columbus ist der Film in weniger als sechs Minuten im Haus. (test, 9/2013)

Die Motoren beschleunigen die Ketten zum Teil auf rasante $70 \mathrm{~km} / \mathrm{h}$ und mehr. (test, 9/2013) 
rekordverdächtig

Rekordverdächtige 38664 Kinder hatten sich [für „Deutschland sucht den Superstar - Kids"] beworben. (OV, 2012-05-05)

Heidelberg wird bis 2017 insgesamt rekordverdächtige 323 Millionen Euro aus der Exzellenzinitiative bekommen. (Reutlinger Generalanzeiger, 2013-06-21 - online)

samtig

Für jede Rasur der Grashalme gibt es außerdem verschiedene Maschinen, je nachdem wie kurz die Rasenfläche sein muss. Das reicht dann von samtigen drei Millimetern rund um die Löcher bis zu recht haarigen 50 Millimetern am Platzrand. (OV,2011-09-15, 11)

sanft

Tiger mit sanften 1030 Watt [...] Die meisten Staubsauger im Test kommen mit weniger als 1400 Watt aus. Dem Vorwerk Tiger VT265 reichen sogar 1030 Watt, um Teppichboden sehr gut zu reinigen. (test, 1/2013)

satt

Satte $\underline{81 \text { Prozent }}$ [der in einer Umfrage eines Kondomherstellers befragten Amerikaner] würden nach Wegen suchen, ihr Sexualleben aufregender zu gestalten. $(\mathrm{OV}$, 2012-07-14)

BMW hat ein Gewichtsproblem. Das 240i Gran Coupé ist satte 242 Kilogramm schwerer als der vergleichbare Mercedes CLS 350. (Auto, Motor und Sport, 20/2012)

schlank

Samsung muss sich hinter den Spezifikationen des Galaxy S3 nicht verstecken: großes 4,8-Zoll-Display mit 720p-Auflösung, Vierkern-CPU, schlanke 8,6 Millimeter Dicke und ein üppiger Akku mit 2.100 Milliamperestunden sind ein mächtiges Paket. (Computer-Bild, online, 2012-05-10)

schlapp

In einer zertifizierten Null-Kilometer-Trattoria [in der die Speisenbestandteile direkt aus der Umgebung stammen] kann man eine Portion Pasta al Pomodoro für

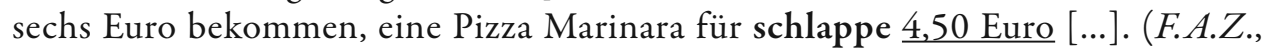
2012-08-20)

\section{schlotternd}

In Deutschland dagegen, wo man in den letzten Tagen in weiten Teilen des Landes Temperaturen von mittelmäßigen 20 und nachts nur schlotternden zehn Grad maß, soll nach einem Bericht des Deutschen Wetterdienstes vom Dienstag die erste Hälfte des Jahres 2011 dennoch zu warm ausgefallen sein - nämlich um 1,5 Grad wärmer, als es hierzulande typisch ist. (F.A.Z., 2011-07-27) 
schmerzhaft

So würde zum Beispiel ein Ölpreisschock mit Preisen zu 150 Dollar je Barrel das Wachstum der Weltwirtschaft um schmerzhafte 2,5 Prozentpunkte bremsen. (Focus Money, Nr. 16/2011, 2011-04-13)

\section{sensationell}

Angeblich liegt die Einschaltquote (die Zahlen sind umstritten) bei sensationellen 30 Prozent. (F.A.Z., 2012-07-10)

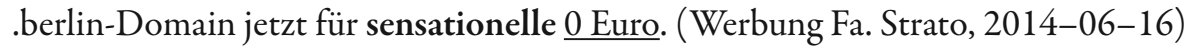

sicher

Sichere $\underline{4,5} \%$ fürs Festgeld (Internet: OnVista, Werbung, 2011-10-01)

spektakulär

Im Dezember 1974 wurde beschlossen, dass die Volljährigkeit und uneingeschränkte Geschäftsfähigkeit auf einen Schlag um spektakuläre drei Jahre gesenkt wurde. (F.A.Z., 2014-03-17)

stattlich

Auch das Gehäuse orientiert sich mit seinem Durchmesser von stattlichen 45 Millimetern (13 Millimeter hoch) an den Vorläufern. (F.A.Z., 2011-08-10)

Allein in der Stadt Frankfurt haben stattliche neun Prozent der zugelassenen Autos [...] keine grüne Plakette [...]. (F.A.Z., 2011-11-30)

Das Kofferraumvolumen beträgt stattliche 645 Liter [...]. (F.A.Z., 2013-10-01)

stolz

Das neue Jahr hat bei Big Dutchman mit zwei ganz besonderen Arbeitsjubiläen begonnen: Heinz Kühling blickt auf stolze 45 Jahre Betriebszugehörigkeit beim Calveslager Stalleinrichter zurück. (OV, 2010-02-06)

Dieser Balsamico ist für stolze 49 Euro bei Manufactum zu haben. (test, 6/2011)

Der sehr gute Haarglätter „Braun Satin Hair 7 “ kostet 63 Euro und das gute Kultgerät "ghd IV styler" stolze 195 Euro. (test, 6/2011)

Nach seinem Referendariat kam er 1977 ans Humboldt-Gymnasium. Das sind mittlerweile stolze 34 Jahre Erfahrung. (SpaZz. Das Magazin für Ulm und Neu-Ulm, Juli 2011)

Ein Testspiel des Zweiligisten FC St. Pauli beim Bezirksligisten Sparta Werlte (4:0) lockte jetzt stolze 2000 Fans ins Werder-Stadion. (OV, 2012-07-09)

Die Verkaufszahlen für Kinderwurst stiegen im Jahr 2010 um stolze 13 Prozent [...]. (test, 9/2012)

Sie [die Kamera] bietet auf Wunsch Dauerbelichtung und eine hohe Empfindlichkeit von stolzen 12800 Iso. (test, 9/2012)

Die Superzoom-Kamera bringt es auf stolze 18 Megapixel. (test, 9/2012) 
sündhaft

Der Traum von der Weltreise, die sündhafte 20000 Euro kostet, dürfte zu den verborgenen Schulden zählen. (F.A.Z., 2012-12-22)

teuer

Die Seminare kosteten 40 bis teure 1400 Euro und dauerten zwischen 7 und 15 Stunden. (test, 6/2011)

\section{überraschend}

In Mecklenburg-Vorpommern sind es 60 Prozent, die über diese Verstehenskompetenz [des Niederdeutschen] verfügen, im Stadtstaat Bremen sind es durchaus überraschende 58 Prozent. (Frerk Möller: Plattdeutsch im 21. Jahrhundert. Bestandsaufnahme und Perspektiven. [...] Leer: Schuster, 2009)

\section{übersichtlich}

[Über Kate Moss:] Kindchenschema und Heroin-Chic, hohe Wangenknochen, kleine Brüste, laszive Lippen und übersichtliche 170 Zentimeter vereinigten sich zu einer Melange mit Sogwirkung. (Zweitausendeins Merkheft 272, Oktober 2013)

uneinholbar

Uneinholbare acht Punkte beträgt zwei Spieltage vor dem Saisonende der Vorsprung [von Borussia Dortmund] auf den schärfsten Verfolger Bayer [Leverkusen]. (OV am Sonntag, 2011-05-01)

unfassbar

Er [= Rainer Werner Fassbinder] drehte bis zu seinem Tod mit 37 Jahren unfassbare 40 Filme [...] (Zweitausendeins: Merkheft 256, Juni 2012)

\section{unfreundlich}

Es ist einer der ersten kühlen Abende in diesem Herbst. Unfreundliche zehn Grad lassen die Bockwürste der Zuschauer des Fußballspiels SC Uchte gegen den BSV Rehden erkalten. (F.A.Z., 2013-11-29)

\section{unglaublich}

In Hufeisenform stürzen hier [an den Niagarafällen] unglaubliche 2,7 Millionen Liter Wasser pro Sekunde auf einer Breite von 670 Metern etwa 54 Meter in die Tiefe. (Reiseprospekt „Kanada: Indian Summer“, Marco Polo Reisen, 2010)

Unglaubliche 850 Meldungen. Ponyclub Dinklage hat an drei Turniertagen rappelvolles Programm (Rundschau am Mittwoch [Vechta], 2011-03-09, Überschrift und Unterüberschrift)

\section{unterhaltsam}

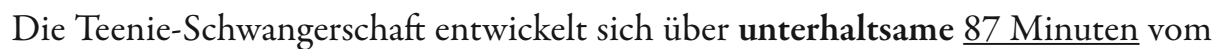
Problem zur Herausforderung - und schließlich zum Glück. (OV, 2014-04-04) 


\section{unvorstellbar}

Rubber Soul - Ab hier zeigten die Beatles ihre wahre Klasse. Zwischen „Help!“ und „Rubber Soul“ muss irgendetwas Umwälzendes passiert sein. Zeitlich lagen nur unvorstellbare 4 Monate zwischen der Veröffentlichung der beiden Alben. Von der musikalischen Entwicklung her, könnten es aber auch 20 Jahre sein. [...] (Kundenrezension von „The Beatles Stereo Box": www.amazon.de)

üppig

Üppige 120 Liter Wasser [...] LG zapft fürs Waschtrocknen üppige 120 Liter aus dem Hahn, AEG immerhin knapp 100 Liter. (test, 10/2012)

\section{verschwindend}

Vor zwölf Jahren mussten noch gut vier Prozent [der Studienabsolventen] diese Zensur [sc. ,ausreichend"] hinnehmen, nun sind es nur noch verschwindende 1,1 Prozent. (Süddeutsche Zeitung, 2012-NOV-10/11: www.sueddeutsche.de)

\section{wahnsinnig}

Mit wahnsinnigen 41 Megapixeln und neuer Technologie soll sie [sc. die Kamera] für gestochen scharfe Bilder sorgen. (test, 10/2012)

\section{warm}

Bei warmen 29 Grad zu Beginn des Spiels hatten sie [sc. die Niederländer] ein paar gute Szenen in der Anfangsphase [...]. (OV, 2012-06-14)

\section{winzig}

Beim Gewehrschießen wird mit Kugeln auf einer Entfernung von 10 Metern bis 50 Metern Abstand geschossen. Das zu treffende Ziel variiert somit zwischen winzigen 0,5 Millimetern und 10,4 Millimetern Durchmesser. (postfrisch, Juli/August 2011)

Winzige 150 Jahre Industriegeschichte werfen die Welt aus der Bahn. (OV, 2014-04-01)

zart

Jungfräulich, betörend schön und zarte 21 [sc. Jahre]: Anastasia Steele ist eine verträumte Literaturstudentin. (Zweitausendeins: Merkheft 263, Januar 2013) 